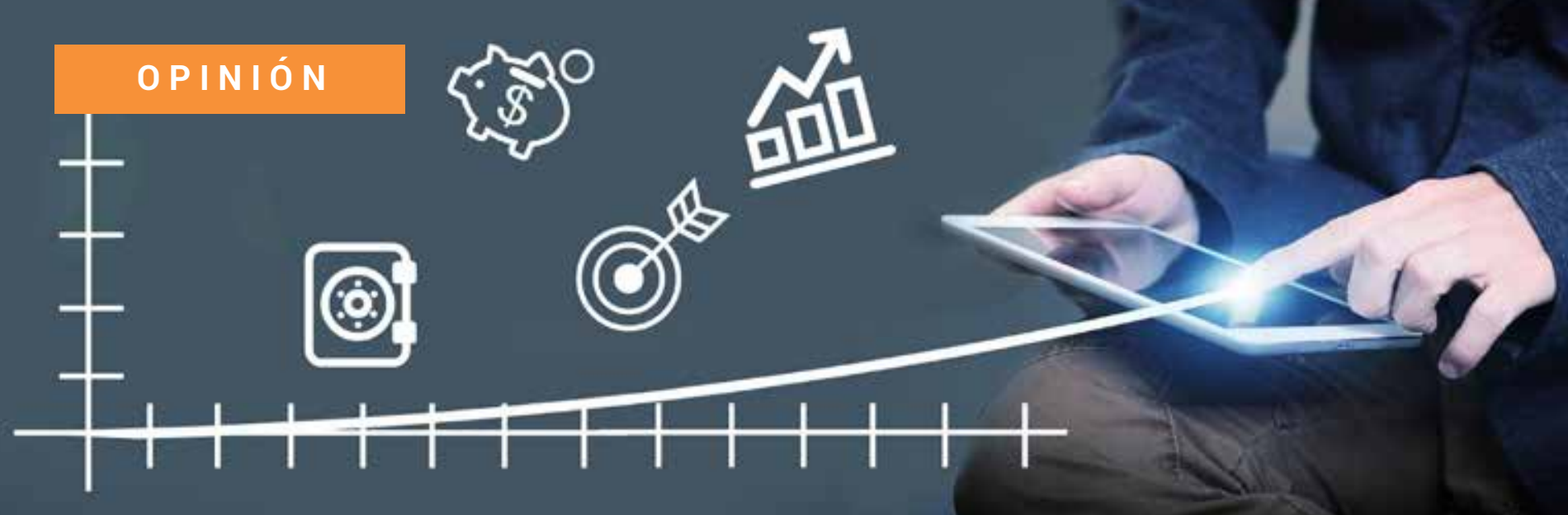

\title{
Influencia de la inteligencia de negocios en los procesos de toma de decisiones dentro de las instituciones financieras
}

\author{
Por. Guillermina Mora \\ Coordinadora de la Licenciatura en Administración de Empresas de la Universidad Iberoamericana Puebla \\ guillermina.mora.basurto@iberopuebla.mx
}

Las nuevas tecnologías de la información y sus diferentes aplicaciones han modificado el ambiente de negocios haciendo que las personas puedan tener acceso a un sinfín de datos e información en tiempo récord y al más bajo costo, desarrollando herramientas que logran analizar datos de mercado junto con información financiera y contable al mismo tiempo (Rakovic, Durkovic \& Trninic, 2011). $\mathrm{La}$ inteligencia de negocios (Business Intelligence, como el término anglosajón) ha empezado a jugar un papel muy importante dentro del desempeño de las empresas y el desarrollo organizacional, ayudándolas a mejorar y establecer ventajas competitivas.

Las empresas que conforman el sector financiero encuentran diferentes desafíos ante la necesidad de definir productos y servicios vanguardistas para sus clientes, y porque esto depende en gran medida de la capacidad de análisis de la información que éstas tengan. Algunos autores han referido que las empresas de este sector se enfrentan a diversas fallas que aparecen durante la gestión de sus estrategias, relacionadas en su mayoría, con la falta de indicadores que informen sus decisiones y la ausencia de un análisis efectivo de la información financiera. Ante este escenario la inteligencia de negocios se presenta como una alternativa para fortalecer e informar las capacidades organizacionales en función de crear ventajas competitivas sostenibles que maximicen su valor.
El sector financiero es una industria que ha tenido un crecimiento sostenido en varios países en los últimos años $\mathrm{y}$, de acuerdo con el Banco Mundial, tiene entre sus objetivos poseer sistemas de finanzas inclusivos. En este contexto, la inteligencia de negocios toma especial protagonismo pues el procesamiento y análisis de grandes cantidades de datos - dos acciones fundamentales - son las que sirven como insumo para el proceso de toma de decisiones al interior de las instituciones. Estas condiciones son relevantes si se considera que los mercados financieros son un aspecto fundamental para el desarrollo y crecimiento de los países, de tal forma que, si operan bajo una base sólida, brindan información fiable y accesible, y disminuyen costos de transacción al canalizar la asignación de recursos para el crecimiento económico (IMCO, 2012).

Como se puede observar, la combinación de la informática y las nuevas tecnologías de la información en las organizaciones significan un elemento estratégico en función de adquirir y mantener una ventaja competitiva; es así, como la inteligencia de negocios se sirve y necesita del acceso a datos e información diariamente, lo que permite a las empresas el análisis de la situación actual de los negocios y su desempeño, lo que significa que la organización tiene la información correcta, en el momento indicado y a disposición de las personas que lo necesitan (Guarda, Santos, Augusto, Silva \& Pinto, 2013). 
Partiendo del concepto de gestión del conocimiento, se acuña el de inteligencia de negocios, el cual ha sido abordado en el área tecnológica y de software que se ofrece a las organizaciones para poder implementar sistemas de información que reforzarán las estrategias y acciones en la administración. Entre varias definiciones se destaca la de Nelson (2010) define a la inteligencia de negocios como "una estrategia administrativa para crear un método más estructurado al tomar decisiones."(p.2).

Estudios como el elaborado por Mobaraki (2017) han constatado que el emplear la información existente permite a la empresa utilizar las ventajas competitivas generadas por el conocimiento en sí mismo y ser pionero al comprender mejor las demandas y necesidades de los clientes, así como facilitar la gestión de las interacciones con ellos para, en la medida de lo posible, controlar los cambios negativos o positivos; debido a lo anterior se debe tener en cuenta que los nuevos desafíos de gestión han aumentado la importancia de los capitales basados en el conocimiento.

La relevancia de los factores humanos bajo un esquema de inteligencia de negocios está condicionada en gran medida por el hecho de que la tecnología de la información por sí sola no puede resolver la variedad de problemas de la integración de la información para cumplir con las expectativas del usuario. La experiencia con las comunidades de inteligencia ha demostrado que el crecimiento sostenido se debe en gran medida a la motivación para compartir y contribuir, explicitando con ello la intención de utilizar la "cultura de la inteligencia" como un término más en el campo de la gestión de la información y su estrecha relación con los factores humanos que convergen en una organización, lo cual a su vez ha generado discusión en los últimos años (Pillar, 2011; Snow, 2014, citados por Skyrius \& Nemitko, 2018).

Cabe acotar que cultura de la información se define como "un subconjunto de la cultura organizacional general en la que el valor y la utilidad de la información para alcanzar el éxito operacional y estratégico forma la base de la toma de decisiones de la organización" (Curry \& Moore, 2003 citado por Popovič, Hackney, Coelho, \& Jaklič, 2014, p. 272). La cultura de la información abarca conductas, normas y valores socialmente compartidos que definirán la importancia, gestión y utilización de la información en una empresa.

La toma de decisiones es el núcleo de la planeación dentro del proceso administrativo, Kościelniak \& Puto (2015) sustentan que en la actualidad el entorno cambia rápidamente, junto con la evolución de la informática, las telecomunicaciones y los medios electrónicos; es por ello, que en este contexto los procesos de toma de decisiones de las empresas se ven impactados pues están acompañados por una gran cantidad de datos y software para su análisis, mismos que deben involucrarse en este proceso de gestión organizacional. Es en este marco que las gerencias y quienes toman decisiones de negocios en las empresas se preguntan cómo utilizar datos masivos de forma eficaz y eficiente.

\section{Discusión}

Las empresas de la industria financiera deben de ser resistentes a los cambios del mercado y a las nuevas regulaciones para seguir teniendo éxito y ser capaces de dar respuesta al mercado y sus nuevas necesidades. La efectividad de las organizaciones converge tanto en la disponibilidad y uso de la información así como también en el aprovechamiento de la tecnología en la toma de decisiones; y es que bajo un esquema de inteligencia de negocios la información es uno de los activos más importantes y por ende el conocimiento generado al utilizar dicha información en procesos organizacionales.

En países desarrollados, a partir de la evolución de las tecnologías de la información y el énfasis en el capital humano capacitado en las mismas para el manejo y transformación de datos, la industria que más se ha visto influenciada por la inteligencia de negocios es la financiera y por ende la que conlleva una mayor experiencia en ese campo. Esto último incide de forma directa en la calidad de los datos que obtienen las personas que manejan las tecnologías; por ello, en la medida en la que se tenga el capital humano con las competencias requeridas, la efectividad de los procesos de la obtención de información y un proceso intencionado e informado de toma decisiones se podrán ver efecto en una mayor competitividad de mercado y mayor eficiencia en los servicios que ofrecen las empresas del sector. 


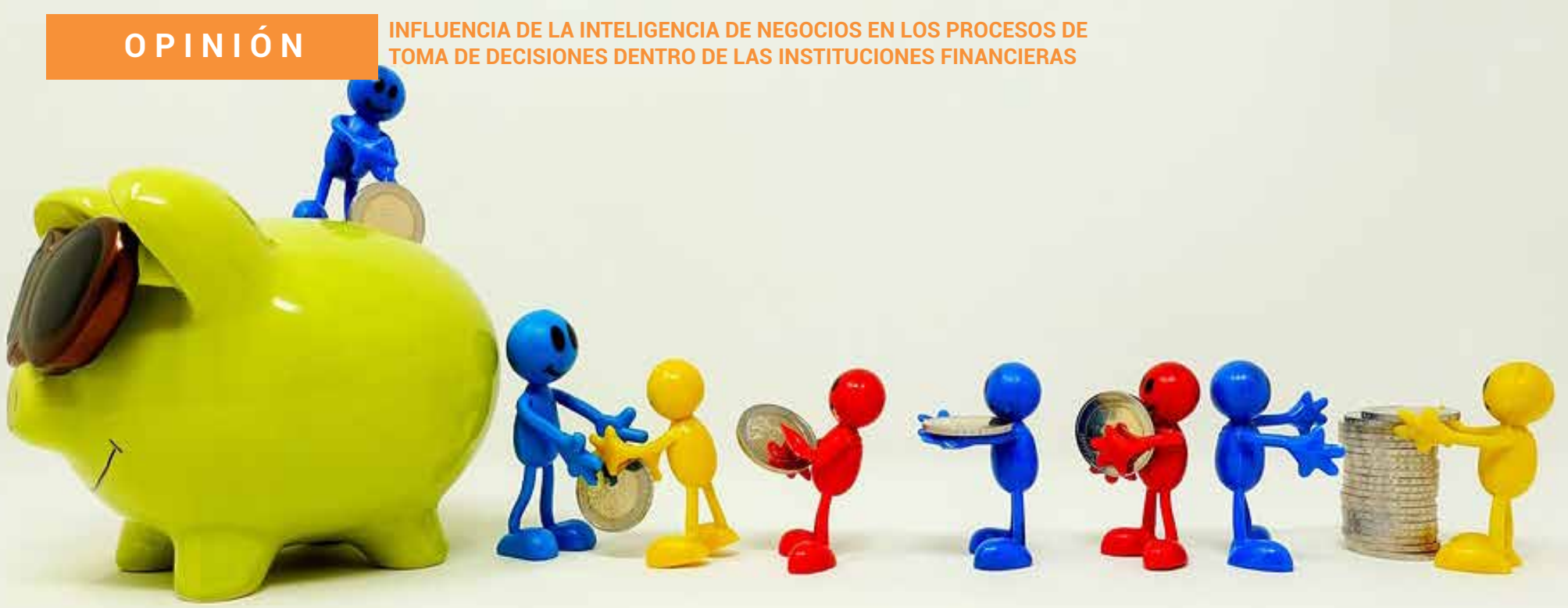

De acuerdo a la información que muestra el sector financiero, se destaca la competencia en el mercado de las Fintech (Tecnologías financieras en su traducción al español), las cuales se han mostrado desafiantes por su disrupción al ofrecer servicios financieros en línea, siendo más eficientes en cuanto a costos; por otro lado, se encuentra su cálculo de riesgos que se da con mayor asertividad al basar sus decisiones en información suficiente y en tiempo real, dado que toda inversión ya sea financiera, empresarial o de seguros, conlleva uno. Otro aspecto relevante de estas tecnologías es el de las regulaciones, las cuales implican cierta complejidad debido a los constantes cambios que se realizan y con la que se debe estar actualizado a la hora de ofrecer el tipo de servicio financiero de acuerdo con el tipo de cliente que se atiende.

La firma Ernest \& Young (EY) realizó en España (octubre 2014) un estudio sobre la banca y los servicios ofrecidos a los clientes en el que se muestran las oportunidades y aplicaciones del Big Data (nombre anglosajón asignado al trabajo de manejo de datos a gran escala), un ejemplo específico sobre cómo usar y procesar grandes cantidades de información. De acuerdo con este estudio, es posible que cada empresa que trabaja bajo estos esquemas tenga amplias posibilidades de generar valor a sus clientes debido a que aprovechan la calidad de los datos en tiempo real que procesan. Sin embargo, y a pesar de contar con este tipo de evidencias y ejemplos, se está empezando a sentir frustración por el poco progreso de la industria al respecto.

$Y$ es que implementar un proyecto de estas magnitudes enmarcado en el modelo de inteligencia de negocios al interior de una empresa es complejo porque necesita, además del convencimiento y anuencia de la alta dirección, una considerable inversión en infraestructura y capacitación del personal involucrado en el proyecto de forma directa en el manejo de datos y de los clientes internos, quienes toman decisiones basados en estos modelos. De ahí que en el citado estudio expongan las principales barreras para trabajar bajo este modelo, entre las cuales señalan las capacidades tecnológicas y la falta de personal competente, pues es necesario contar con profesionales como "científicos de datos, expertos en visualización, analistas de negocio, profesionales en la gestión de datos y otros especialistas como expertos en el tratamiento de datos confidenciales" (EY, 2014, p. 16). Ante este panorama, la industria financiera se perfila como una con los mejores potenciales para tener un proyecto que involucre este tipo de tecnología con las personas más competentes para ello.

De acuerdo con Moro, Cortez y Rita (2015) las iniciativas de inteligencia de negocios tienen un mayor auge en las empresas del sector financiero, en particular por la predicción de riesgo en función de la aprobación o negación de los créditos a clientes; igualmente, existe un interés importante en la posibilidad de identificar a través de estas herramientas la propensión a una quiebra y la predicción de fraude.

Por otro lado, la banca también se enfoca en la retención de sus clientes con el ofrecimiento de productos hechos a su medida y con un alto grado de certidumbre en función de la inversión y los instrumentos para tal efecto. La banca electrónica oferta una amplia variedad de servicios, los cuales podrían implicar tareas no transaccionales como la visualización de saldos u operaciones recientes; o demandas de transacciones reales como las transferencias de los fondos, facturas, pagos, inversiones en acciones y bonos, etc. Las soluciones de banca móvil son hoy en día un gran desafío para el sector financiero debido a que pretenden adaptar su enfoque a nuevos clientes que utilizan dispositivos electrónicos como celulares inteligentes, tabletas, etc. 


\section{Conclusiones}

Los países de América Latina en específico se perfilan como clientes potenciales para este tipo de iniciativas, las cuales afectarán de forma sustancial la competitividad en el sector de servicios financieros de la región. Es momento de eliminar barreras para emprender proyectos de tecnologías disruptivas y de vanguardia como el que presenta el esquema de inteligencia de negocios y el manejo de datos a gran escala; donde el elemento humano competente será el más valorado por la organización, pues son esquemas basados en la gestión de la información y la generación de modelos prospectivos que se aprovecharán para la oferta de servicios mucho más asertivos de acuerdo a cada tipo de clientes que tiene y que podría tener acceso a los servicios financieros.

Las empresas de este sector deben ser quienes lideren esta disrupción hacia el tratamiento de datos y la gestión de riesgo, donde además se dispone de generaciones que se van a adaptar a esta nueva forma de trabajo por su propensión a las herramientas tecnológicas y su evolución. Para ello se necesitará de una ardua capacitación y verdadero cambio de paradigma y de formas de trabajar, donde posiciones dentro de las empresas terminarán en la extinción, surgiendo otras que se basan puramente en el manejo y análisis de datos.

\section{Bibliografia:}

American Chamber/México, IMCO (2012), Crecimiento y profundización del sector financiero en México. Recuperado de: http://imco.org.mx/wp-content/uploads/2012/7/estudio_sector_financiero. pdf

EY (2014) Big Data en el sector financiero español: Resultados de la encuesta sectorial sobre Big Data. Recuperado de: https:// www.ey.com/Publication/vwLUAssets/EY-big-data-en-el-sector-fi nanciero-espanol/ SFILE/EY-big-data-en-el-sector-financiero-espanol.pd $f$

Guarda, T., Santos, M. F., Augusto, M. F., Silva, C., \& Pinto, F. (2013). Process Mining: A framework proposal for Pervasive Business Intelligence. Information Systems and Technologies (CISTI), 2013 8th Iberian

Conference on (1-4). IEEE. Recuperado de: http://web.b.ebscohost.com/ehost/pdfviewer/pdfviewer?sid=3cf56659-c932 $-4 c 9 a-8 a 1 b-c b 803 d f 25595 \% 40$ sessionmgr715\&vid=4\&hid $=128$

Kościelniak, H., \& Puto, A. (2015). BIG DATA in Decision Making Processes of Enterprises. Procedia Computer Science, 65, 1052-1058. Recuperado de: http://ac.els-cdn.com/S1877050915028835/1-s2.0-S1877050915028835-main.pdf?_ti$d=b 79892 c 8-2 a a 1-11$ e6-94d5-00000aabOf6b\&acdnat=146507 8654_65719fddcc8d425270ea00c41bc03ae8

Mobaraki, E. (2017). A Model for Improving the Business Intelligence of the Companies Envisaging Knowledge Management Approach: A Case Study of the Knowledge-Based Organizations of East Azarbaijan Science and Technology Park. Journal of Engineering and Applied Sciences, 12(19), 5024-5034. Recuperado de: http://docsdrive.com/pdfs/medwelljournals/jeasci/2017/5024-5034.pdf

Moro, S., Cortez, P., \& Rita, P. (2015). Business intelligence in banking: A literature analysis from 2002 to 2013 using text mining and latent Dirichlet allocation. Expert Systems with Applications, 42(3), 1314-1324. Recuperado de: https://ac.els-cdn.com/S0957417414005636/1-s2.0-S095741741400563

6-main.pdf?_tid=d4a16331-ed09-424e-b9a9-52ffc375cb8c\&acdnat=1550 520344_5457242ca7d224c63173a5cfec3f9499

Nelson, G. S. (2010). Business Intelligence 2.0: Are we there yet. SAS Global Forum 2010 Recuperado de: http://www.pharmasug.org/cd/papers/MA/MA07.pdf

Popovič, A., Hackney, R., Coelho, P. S., \& Jaklič, J. (2014). How information-sharing values influence the use of information systems: An investigation in the business intelligence systems context. The Journal of Strategic Information Systems, 23(4), 270283. Recuperado de: https://ac.els-cdn.com/S0963868714000316/1-s2.0-S09638687140003 16-main.pdf?_tid=68571b19-cf024dda-a77d-246898d78961\&acdnat= 1 532558552_e971e52e38e6d3b9914df284398f9d30

Rakovic, L., Durkovic, J., \& Trninic, J. (2011). Business intelligence as support to knowledge management. Perspectives of Innovations, Economics and Business, PIEB, 2 (8), 35-40. Recuperado de: http://academicpublishingplatforms.com/downloads/pdfs/ pieb/volume8/201 106151256_06_PIEB_V8_RS_JelicaTrninic_et_al_Business_Intelligence. pdf

Skyrius, R., \& Nemitko, S. (2018). The Support of Human Factors for Encompassing Business Intelligence. Preceedings of the Informing Science + Information Technology Education Conference Recuperado de: http://proceedings.informingscience.org/InSITE2018/InSITE2018p021-03 4Skyrius4527.pdf 